\title{
Associations of Cardiovascular and Non-Cardiovascular Comorbidities with Dementia Risk in Patients with Diabetes: Results from a Large UK Cohort Study
}

\author{
B. Zheng 1 , B. Su ${ }^{2}$, C. Udeh-Momoh ${ }^{1}$, G. Price ${ }^{1}$, I. Tzoulaki $i^{2,3,4}$, E.P. Vamos ${ }^{6}$, A. Majeed ${ }^{6,7}$, E. Riboli ${ }^{2,7}$, \\ S. Ahmadi-Abhari ${ }^{1}$, L.T. Middleton ${ }^{1,7}$ \\ 1. Ageing Epidemiology Research Unit, School of Public Health, Imperial College London, London, UK; 2. Department of Epidemiology and Biostatistics, School of \\ Public Health, Imperial College London, London, UK; 3. BHF Centre of Excellence, Imperial College London, London, UK; 4. Dementia Research Institute, Imperial \\ College London, London, UK; 5. Hygiene and Epidemiology, University of Ioannina, Ioannina, Greece; 6. Department of Primary Care and Public Health, School of \\ Public Health, Imperial College London, London, UK; 7. Public Health Directorate, Imperial College NHS Healthcare Trust, London, UK
}

Corresponding Author: Prof. Lefkos Middleton, Ageing Epidemiology Research Unit, School of Public Health, Faculty of Medicine, Imperial College London, London W6 8RP, UK. E-mail: 1.middleton@imperial.ac.uk; Tel: +44 203311 0216; Fax: +44 2033110216.

\begin{abstract}
BACKGROUND: Type 2 diabetes (T2D) is an established risk factor for dementia. However, it remains unclear whether the presence of comorbidities could further increase dementia risk in diabetes patients.

OBJECTIVES: To examine the associations between cardiovascular and non-cardiovascular comorbidities and dementia risk in $\mathrm{T} 2 \mathrm{D}$ patients.

DESIGN: Population-based cohort study.

SETTING: The UK Clinical Practice Research Datalink (CPRD).

PARTICIPANTS: 489,205 T2D patients aged over 50 years in the UK CPRD.

MEASUREMENTS: Major cardiovascular and noncardiovascular comorbidities were extracted as time-varying exposure variables. The outcome event was dementia incidence based on dementia diagnosis or dementia-specific drug prescription.

RESULTS: During a median of six years follow-up, 33,773 $(6.9 \%)$ incident dementia cases were observed. Time-varying Cox regressions showed T2D patients with stroke, peripheral vascular disease, atrial fibrillation, heart failure or hypertension were at higher risk of dementia compared to those without such comorbidities (HR [95\% CI] = 1.64 [1.59-1.68], 1.37 [1.34-1.41], 1.26 [1.22-1.30], 1.15 [1.11-1.20] or 1.10 [1.03-1.18], respectively). Presence of chronic obstructive pulmonary disease or chronic kidney disease was also associated with increased dementia risk (HR [95\% CI] = 1.05 [1.01-1.10] or 1.11 [1.07-1.14]).

CONCLUSIONS: A range of cardiovascular and noncardiovascular comorbidities were associated with further increases of dementia risk in T2D patients. Prevention and effective management of these comorbidities may play a significant role in maintaining cognitive health in T2D patients.
\end{abstract}

Key words: Type 2 diabetes, dementia, comorbidity, cohort.

\section{Introduction}

A lzheimer's disease (AD) and other forms of late-onset dementia (LOD) are fast becoming major healthcare and socio-economic challenges across the globe, in parallel with increases in life expectancy and population ageing $(1,2)$. Type 2 diabetes (T2D) is an established risk factor for dementia and is associated with a 53\%-73\% higher risk of LOD or AD (3-5). We previously showed that high and unstable glycaemia, and acute or microvascular diabetic complications are associated with increased dementia risk among older T2D patients (6), highlighting the importance of effective diabetes management in maintaining cognitive health.

The presence of comorbidities is another clinical challenge in diabetes management. Comorbidities, especially cardiovascular diseases (7), are common in T2D patients. Based on a cross-sectional study of 9832 T2D patients across 13 countries, the authors reported that over $30 \%$ of patients had prevalent cardiovascular diseases (7). The aim of the current study was to explore and quantify potential effects of the presence of common, chronic diseases of ageing on dementia risk, in T2D patients.

In this regard, we have comprehensively evaluated the longitudinal associations between a diverse range of major cardiovascular and non-cardiovascular comorbidities and the risk of incident dementia in a cohort of T2D patients, aged over 50 years, leveraging electronic health record (EHR) data from the UK Clinical Practice Research Datalink (CPRD) (8).

\section{Methods}

\section{Data sources}

The UK CPRD GOLD database (8) is a longitudinal national primary care database that includes EHR data 
of over 17 million individuals, currently or previously registered with over 700 general practitioner (GP) practices in the UK. CPRD has also been linked to secondary care data (Hospital Episode Statistics, HES), mortality data from the Office for National Statistics (ONS) and area-based data on measures of social deprivation. The CPRD population has the same profile regarding age, sex and ethnicity as the general population of the UK (8).

\section{Study population}

Participants were included if they were aged 50 years or over at any point during their CPRD registration period between 1987 and 2018 and had a diagnosis of diabetes, based on relevant CPRD Medcode or a prescription of anti-diabetes drugs (oral hypoglycaemic agents or insulin) (6). In addition, eligible patients should have been registered in CPRD for at least one year before diabetes onset to allow time for baseline information to be recorded and to ensure that the date of newly diagnosed diabetes was captured. Patients with a diagnosis of type 1 diabetes, or those who had a diagnosis of diabetes or initiation of anti-diabetic treatment before the age of 30 were excluded. Patients who had a diagnosis of dementia before cohort entry were also excluded. A total of 489,205 individuals were included in the analysis.

\section{Exposure assessment}

Records of six cardiovascular comorbidities (coronary heart disease, stroke, atrial fibrillation, heart failure, peripheral vascular disease and hypertension) and three major non-cardiovascular comorbidities (chronic kidney disease, chronic obstructive pulmonary disease [COPD] and cancer) were extracted using the corresponding CPRD Medcode and Enttype code. These comorbidities were selected as they are common chronic diseases and leading causes of death and disability in older adults (9). To comprehensively identify hypertensive cases, we additionally used blood pressure recordings (systolic blood pressure $\geq 140 \mathrm{mmHg}$ or diastolic blood pressure $\geq 90 \mathrm{mmHg}$ ). The date of onset of a specific type of comorbidity was defined according to its first relevant health record. The date of onset of overall cardiovascular comorbidities was defined as the earliest date of developing one of the six comorbidities mentioned above.

In addition, information on the following covariates at cohort entry was extracted: age, sex, calendar year of cohort entry, region in the UK, quintiles of index of multiple deprivation (IMD, a proxy of socio-economic status), body mass index (BMI), smoking status, duration of diabetes (based on the first clinical record of diabetes diagnosis), history of prescription of anti-diabetes medications and recorded diabetic complications.

\section{Outcome ascertainment}

The outcome event was dementia incidence. Patients were considered to have dementia if they had: 1) a dementia diagnosis based on Medcode in CPRD; 2) a dementia diagnosis based on ICD codes in linked HES or ONS records; or 3) at least one dementia-specific drug prescription (donepezil, galantamine, rivastigmine or memantine) (6). Among the extracted dementia cases, $96 \%$ were based on diagnosis codes and $4 \%$ were based on dementia-specific drug record. The outcome event date was defined as the date of the first dementia diagnosis or the first prescription date of dementiaspecific drugs, whichever occurred earlier.

\section{Statistical analyses}

Distributions of baseline characteristics were summarised and compared between patients with and without cardiovascular disease (except hypertension) at baseline. Time-varying Cox proportional hazards models, with age as the time-scale, were used to estimate hazard ratios (HR) and 95\% confidence intervals (CI) of dementia associated with comorbidities. The presence of six cardiovascular comorbidities in aggregate and by type, as well as three non-cardiovascular comorbidities, were treated as time-varying variables and examined in separate Cox models. Patients with newly developed comorbidities during follow-up contributed person-years to the no comorbidity group up until the comorbidity diagnosis date and then contributed person-years to the comorbidity group. For each patient, time of cohort entry was defined as the date of diabetes onset, aged 50 or January 1, 1987, whichever was the latest. The end of follow-up was defined as the date of dementia incidence, death, transfer out date, last data collection date of the GP practice or May 1, 2018, whichever occurred first.

To account for potential confounding biases, three sequential models with increasing levels of covariate adjustment were created for all analyses: Model 1 only adjusted for age, sex, calendar year and region; Model 2 additionally adjusted for IMD (quintiles), smoking status (non-smoker, current smoker, ex-smoker or missing), BMI category $\left(<25,25\right.$ to $<30, \geq 30 \mathrm{~kg} / \mathrm{m}^{2}$ or missing), and history of comorbidities (for mutual adjustment); Model 3 additionally adjusted for diabetes-related factors, including the duration of diabetes, $\mathrm{HbA1c}$ level, presence of diabetic complications (including hypoglycaemia) and prescription of anti-diabetes drugs (no drug, only oral hypoglycaemic drug, or insulin). Covariates that could change over time (e.g., HbA1c level and status of diabetic complications) were also modelled as timevarying variables and updated at comorbidity diagnosis date during follow-up. Missing values of BMI category and smoking status during follow-up were imputed with the last observation carried forward.

The statistical analyses were conducted using Stata 
Table 1. Baseline characteristics of diabetes cohort by presence of cardiovascular disease

\begin{tabular}{|c|c|c|c|}
\hline \multirow[t]{2}{*}{ Characteristics } & \multirow[t]{2}{*}{ Full cohort } & \multicolumn{2}{|c|}{ Cardiovascular disease at baseline ${ }^{*}$} \\
\hline & & No & Yes \\
\hline Number of participants & 489,205 & 325,168 & 164,037 \\
\hline Baseline age (mean), year & 65.2 & 63.5 & 68.5 \\
\hline Year of cohort entry (median) & 2007 & 2006 & 2008 \\
\hline Obesity $\left(\mathrm{BMI} \geq 30 \mathrm{~kg} / \mathrm{m}^{2}\right), \%$ & 41.4 & 41.6 & 40.8 \\
\hline Current smoker, $\%$ & 19.1 & 18.8 & 19.6 \\
\hline Ex-smoker, \% & 35.1 & 32.3 & 40.3 \\
\hline CKD, \% & 6.3 & 4.1 & 10.7 \\
\hline Ever use of anti-diabetes drugs, $\%$ & 17.4 & 18.2 & 15.7 \\
\hline Baseline HbA1c level (mean), \% & 7.4 & 7.5 & 7.2 \\
\hline Presence of diabetic complications, $\%$ & 2.3 & 2.2 & 2.4 \\
\hline
\end{tabular}

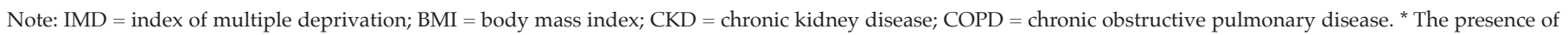

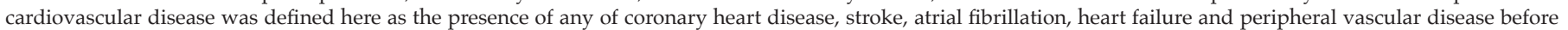

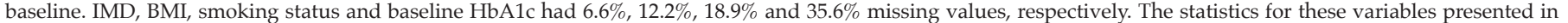
this table are based on complete cases.

(version 15, Stata). All statistical tests were two-sided, and the significance level was $\mathrm{P}<0.05$.

\section{Results}

\section{Baseline characteristics of study population}

Of the 489,205 patients with type 2 diabetes, $52.0 \%$ were male; the mean baseline age was $65.2(\mathrm{SD}=11.2)$ years (Table 1 ). At cohort entry, $41.4 \%$ of patients had obesity (BMI $\geq 30 \mathrm{~kg} / \mathrm{m}^{2}$ ) and $19.1 \%$ or $35.1 \%$ were self-reported current or ex-smokers; $17.4 \%$ had been prescribed anti-diabetes medications, and the mean baseline $\mathrm{HbA} 1 \mathrm{c}$ level was $7.4 \%$. Before baseline, 84,702 $(17.3 \%), 35,808(7.3 \%), 415,197(84.9 \%), 28,270(5.8 \%)$, $20,069(4.1 \%)$ and $56,918(11.6 \%)$ patients already had coronary heart disease, stroke, hypertension, atrial fibrillation, heart failure and peripheral vascular disease, respectively. There were 20,324 $(4.2 \%), 30,881(6.3 \%)$ and $48,940(10.0 \%)$ patients with COPD, chronic kidney disease and cancer before baseline.

Compared to T2D patients without cardiovascular disease (not including hypertension) at baseline, those with pre-existing cardiovascular disease were slightly older, more socio-economically deprived, and more likely to be male, current or ex-smokers and have more noncardiovascular comorbidities $(\mathrm{P}<0.05)$.

\section{Cardiovascular comorbidities in associations with dementia risk}

During a median of 6 years follow-up (ranging from $0-31$ years) of the 489,205 T2D patients, 33,773 (6.9\%) incident dementia cases were recorded. During the follow-up, 38,148 (7.8\%), 27,232 (5.6\%), 44,620 (9.1\%), $27,615(5.6 \%), 23,394(4.8 \%)$ and $75,258(15.4 \%)$ patients developed coronary heart disease, stroke, hypertension, atrial fibrillation, heart failure and peripheral vascular disease, respectively, before dementia diagnosis or censoring.

After adjusting for a full set of covariates (Model 3), there were significant associations of stroke, peripheral vascular disease, atrial fibrillation, heart failure and hypertension with higher risk of dementia incidence (HR $[95 \% \mathrm{CI}]=1.64$ [1.59-1.68], 1.37 [1.34-1.41], 1.26 [1.221.30], 1.15 [1.11-1.20] and 1.10 [1.03-1.18], respectively; Table 2). The HR estimates were even higher in Model 1 and Model 2 with fewer covariates $(\mathrm{P}<0.05)$. In contrast, the presence of coronary heart disease was not robustly associated with dementia risk in Model $3(\mathrm{P}=0.085)$, while Model 1 and 2 revealed a modest association (Table 2). Having any cardiovascular comorbidity was associated with an additional $25 \%$ increase of dementia risk in T2D patients (95\% CI: 15\%-36\%; Table 2). 
Table 2. Associations between presence of cardiovascular and non-cardiovascular comorbidities and risk of dementia among 489,205 diabetes patients

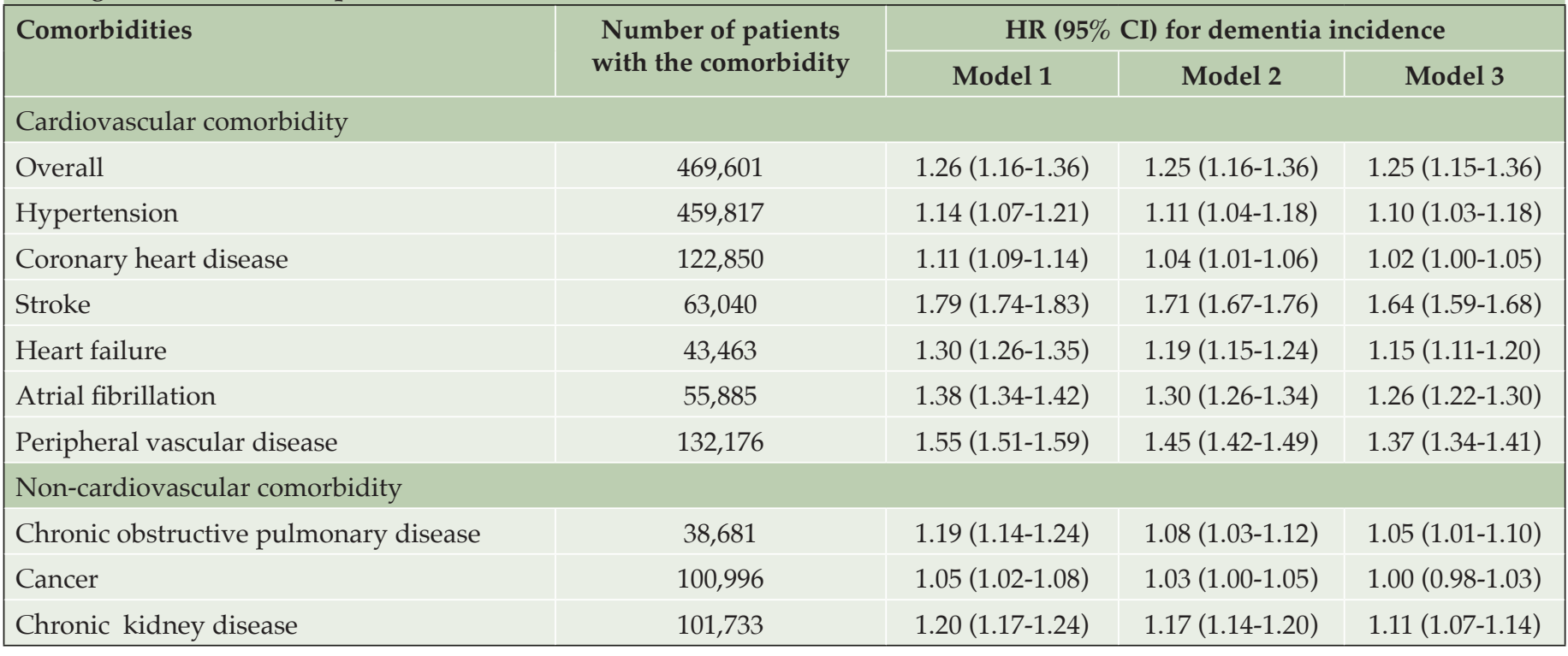

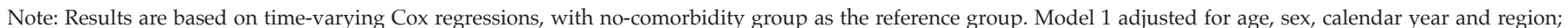

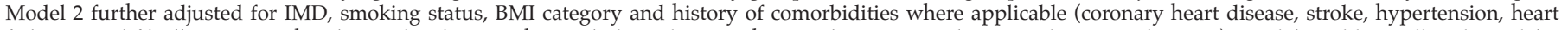

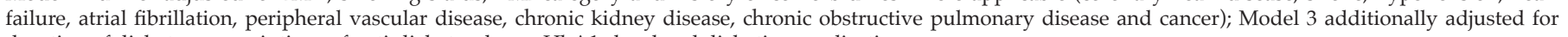
duration of diabetes, prescriptions of anti-diabetes drugs, HbA1c level and diabetic complications.

\section{Non-cardiovascular comorbidities in associations with dementia risk}

Among the non-cardiovascular comorbidities evaluated, $18,357(3.8 \%), 70,852(14.5 \%)$ and 52,056 $(10.6 \%)$ patients developed COPD, chronic kidney disease and cancer during follow-up, respectively. The presence of COPD or chronic kidney disease in T2D patients resulted in a 5\% (95\% CI: $1 \%-10 \%)$ or $11 \%$ (95\% CI: 7\%-14\%) increased risk of dementia incidence, respectively, in the fully adjusted model (Table 2); the magnitudes of these associations were higher in Model 1 and Model 2 with fewer covariates $(\mathrm{P}<0.05)$. In contrast, the presence of cancer was not associated with dementia risk in T2D patients ( $\mathrm{HR}=1.00,95 \%$ CI: 0.98-1.03).

\section{Discussion}

This study is the largest cohort study to date $(489,205$ T2D patients and 33,773 incident dementia cases) that has attempted to explore and quantify the associations of cardiovascular and non-cardiovascular comorbidities with dementia risk, in T2D patients. After taking into account the time-varying nature of the presence of comorbidities and adjusting for a large set of potential confounding factors in the time-varying Cox regressions, we found that a range of cardiovascular and noncardiovascular comorbidities in T2D patients were associated with further increases of dementia risk, among which stroke and peripheral vascular disease are the strongest risk factors.

Our results showing increased dementia risk in T2D patients with cardiovascular comorbidities are in line with previous evidence (10). Exalto et al. (10) reported a summary risk score for prediction of 10-year dementia risk in type 2 diabetes patients based on data from two cohorts ( $\mathrm{n}=29,961$ and 2413). In their prediction model, the presence of cerebrovascular disease $(\mathrm{HR}=1.65,95 \%$ CI: 1.50-1.82) and the presence of cardiovascular disease $(\mathrm{HR}=1.21,95 \% \mathrm{CI}: 1.13-1.29)$ were among the strongest predictors for dementia in T2D patients. Our study provided more comprehensive evidence by investigating the independent associations of different subtypes of cardiovascular comorbidities. The strong association between stroke and dementia risk may be attributed, at least partially, to vascular dementia (11). Stroke may also contribute to the pathogenesis of other LOD forms such as $\mathrm{AD}(12,13)$; furthermore, it is well established that the majority of LOD patients, over the age of 75 , harbour mixed cerebral pathologies $(13,14)$. Unlike stroke, the presence of peripheral vascular disease, atrial fibrillation and heart failure is likely to merely represent peripheral markers of cerebrovascular pathology $(12,13)$. Our finding of diminished association between coronary heart disease and dementia after adjusting for more covariates implies that the association is not independent and could be driven by other risk factors.

This study also showed that hypertension was associated with $10 \%$ increased dementia risk in T2D patients. In fact, the 2020 Report of the Lancet Commission for dementia prevention, intervention and care (15) has identified hypertension and diabetes amongst 12 modifiable risk factors for dementia that could be directly implicated in the neurodegenerative process. The Lancet report has highlighted the potential 
of prevention strategies for dementia. On the other hand, a pioneering randomised control trial (RCT) of multidomain lifestyle intervention, the "Finnish Geriatric Intervention Study to Prevent Cognitive Impairment and Disability" (FINGER) trial (16), showed that a 2-year multidomain intervention of diet, physical activity, cognitive training and management/monitoring of vascular/metabolic risk had efficacy in improving memory and cognition in at-risk older adults.

In contrast, non-cardiovascular comorbidities in T2D patients have drawn less attention with respect to dementia risk. The underlying mechanisms linking COPD and chronic kidney disease with increased dementia risk (e.g., microvascular pathologies or chronic inflammation (17)) warrant further investigation. Future well-powered clinical studies, with deep phenotyping and biomarkerbased characterisation of dementia patients, may help to elucidate the precise contributions of these comorbidities in the pathogenesis of LOD. Previous studies showed that stroke and hypertension were associated with increased risk for dementia (HR for stroke $=1.69,95 \%$ CI: 1.49 1.92 (18); relative risk, RR for hypertension $=1.20,95 \%$ CI: 1.06-1.35 (19)) in the general population regardless of the presence of diabetes, whereas evidence for COPD and chronic kidney diseases was scarce. Whether these diseases and T2D influence dementia risk independently or interactively (e.g., through a synergistic effect) warrants further research.

The incidence rate of dementia among T2D patients in our study (mean age 65.2 years) was 9 cases per 1000 person-years, which is higher than other studies in the general population that also used data from primary care electronic health records. For example, a study of the UK adults aged over 60 years in The Health Improvement Network database reported a dementia incidence rate of 3-4/1000 person-years between 1997 and 2007 (20); another study using the same database (median age 59.5 years) reported an incidence rate of 5.65/1000 personyears in 2015 (21). This reflected the fact that T2D patients are more likely to develop dementia $(22,23)$. A recent review on T2D and cognitive dysfunction (24) pointed out that the screening and diagnosis of cognitive impairment and dementia is important for older adults with T2D, and suggested that future clinical research is warranted to evaluate the role of comorbidities associated with diabetes in increasing risk of cognitive dysfunction. The findings of our study provide evidence on the role of comorbidities in further increasing the risk of cognitive decline and dementia in T2D patients. Their appropriate management, including lifestyle modification, might have a preventative effect on dementia risk in clinical practice.

There are several limitations of this study to be considered in interpreting our results. Firstly, dementia cases could be underreported or under-diagnosed in the CPRD database. Nevertheless, we maximised the dementia case detection using the linked data from HES and ONS databases (secondary diagnosis and cause of death) and dementia-specific drug records. We have also controlled for calendar year in all analyses to account for the increasing diagnosis rate of dementia and changes in dementia diagnostic criteria over time (25). In addition, we did not distinguish by specific types of LOD, as such granular level of data is variably registered in CPRD and the precise coding boundaries and heterogeneity of these dementia types still remain poorly defined $(13,14)$. Although we aimed to capture a broad age range of patients with diabetes (26) to increase the representativeness of this study, future studies focused on older elderly populations with higher dementia incidence rate and possibly different comorbidity profiles are needed. Furthermore, the possibility of residual confounding cannot be excluded. For example, there is little information on education level and on physical and social activities in the CPRD database, which are known risk factors for dementia (15). Finally, although we identified several strong and robust associations, causality cannot be established due to the observational nature of this study.

In conclusion, this large-scale cohort study showed that the evaluated cardiovascular and non-cardiovascular comorbidities, with the exception of coronary heart disease and cancer, are independent risk factors for dementia incidence in patients with T2D. In the absence of disease-modifying therapies for late-onset dementia (27) and given the need to mitigate the increased risk for dementia in people with diabetes, the prevention and effective management of these comorbidities may have a significant role in maintaining cognitive health and reducing the dementia burden among older adults with diabetes.

Funding: This report is based on independent research supported by Diabetes UK (Grant No: 18/0005851) and the National Institute for Health Research (NIHR) Applied Research Collaboration (ARC) Northwest London. The views expressed in this publication are those of the authors and not necessarily those of Diabetes UK and the National Institute for Health Research or the Department of Health and Social Care. The sponsors had no role in the design and conduct of the study; in the collection, analysis, and interpretation of data; in the preparation of the manuscript; or in the review or approval of the manuscript.

Data availability: This study is based on data from the Clinical Practice Research Datalink obtained under license from the UK Medicines and Healthcare Products Regulatory Agency (protocol approved by the Independent Scientific Advisory Committee: No. 19_065R). According to the UK Data Protection Act, information governance restrictions (to protect patient confidentiality) prevent data sharing via public deposition. Data extracts can be requested by applying to the Clinical Practice Research Datalink (https: / / www.cprd.com).

Conflict of Interest Disclosure: L T Middleton has received research funding (to Institution) from Janssen, Merck (USA), Gates, Takeda/ Millenium, Novartis, Invincro and EIT Health, outside the submitted work. All other authors declare no conflict of interest.

Open Access: This article is distributed under the terms of the Creative Commons Attribution 4.0 International License (http:/ / creativecommons.org/ licenses/by/4.0/), which permits use, duplication, adaptation, distribution and reproduction in any medium or format, as long as you give appropriate credit to the original author(s) and the source, provide a link to the Creative Commons license and indicate if changes were made.

\section{References}

1. Alzheimer's Association. 2020 Alzheimer's disease facts and figures Alzheimers Dement. 2020;16:391-460. DOI: https:/ / doi.org/10.1002/alz.12068 
2. Ahmadi-Abhari S, Guzman-Castillo M, Bandosz P, et al. Temporal trend in dementia incidence since 2002 and projections for prevalence in England and Wales to 2040: modelling study. BMJ. 2017;358:j2856. DOI: https://doi. org / 10.1136/bmj.j2856

3. Gudala K, Bansal D, Schifano F, Bhansali A. Diabetes mellitus and risk of dementia: A meta-analysis of prospective observational studies. J Diabetes Investig. 2013;4(6):640-650. DOI: https: / / doi.org/10.1111/jdi.12087

4. Zhang J, Chen C, Hua S, et al. An updated meta-analysis of cohort studies: Diabetes and risk of Alzheimer's disease. Diabetes Res Clin Pract. 2017;124:4147. DOI: https: / / doi.org/10.1016/j.diabres.2016.10.024

5. Chatterjee S, Peters SAE, Woodward M, et al. Type 2 Diabetes as a Risk Factor for Dementia in Women Compared With Men: A Pooled Analysis of 2.3 Million People Comprising More Than 100,000 Cases of Dementia. Diabetes Care. 2016;39(2):300-307. DOI: https: / / doi.org/10.2337/ dc15-1588

6. Zheng B, Su B, Price G, Tzoulaki I, Ahmadi-Abhari S, Middleton L. Glycemic Control, Diabetic Complications, and Risk of Dementia in Patients With Diabetes: Results From a Large U.K. Cohort Study. Diabetes Care. 2021;dc202850. DOI: https: / / doi.org/10.2337/dc20-2850

7. Mosenzon O, Alguwaihes A, Leon JLA, et al. CAPTURE: a multinational, cross-sectional study of cardiovascular disease prevalence in adults with type 2 diabetes across 13 countries. Cardiovasc Diabetol. 2021;20(1):154. DOI: https: / / doi.org/10.1186/ s12933-021-01344-0

8. Herrett E, Gallagher AM, Bhaskaran K, et al. Data Resource Profile: Clinical Practice Research Datalink (CPRD). Int J Epidemiol. 2015;44(3):827-836. DOI https: / / doi.org/10.1093/ije/ dyv098

9. National Center for Chronic Disease Prevention and Health Promotion Chronic Diseases in America. https://www.cdc.gov/chronicdisease/ resources/infographic/chronic-diseases.htm. Accessed 6 September 2021.

10. Exalto LG, Biessels GJ, Karter AJ, et al. Risk score for prediction of 10 year dementia risk in individuals with type 2 diabetes: a cohort study. Lancet Diabetes Endocrinol. 2013;1(3):183-190. DOI: https:/ / doi.org/10.1016/S2213 8587(13)70048-2

11. Ott A, Stolk RP, van Harskamp F, Pols HA, Hofman A, Breteler MM. Diabetes mellitus and the risk of dementia: The Rotterdam Study. Neurology. 1999;53(9):1937-1942. DOI: https: / / doi.org/10.1212/WNL.53.9.1937

12. Sonnen JA, Larson EB, Brickell K, et al. Different Patterns of Cerebral Injury in Dementia With or Without Diabetes. Arch Neurol. 2009;66(3):315-322. DOI: https: / / doi.org/10.1001/archneurol.2008.579

13. Toledo JB, Arnold SE, Raible K, et al. Contribution of cerebrovascular disease in autopsy confirmed neurodegenerative disease cases in the National Alzheimer's Coordinating Centre. Brain. 2013;136:2697-2706. DOI: https: / doi. org/10.1093/brain/awt188

14. Schneider JA, Arvanitakis Z, Bang W, Bennett DA. Mixed brain pathologies account for most dementia cases in community-dwelling older persons. Neurology. 2007;69(24):2197-2204. DOI: https:// doi.org/10.1212/01. wnl.0000271090.28148.24
15. Livingston G, Huntley J, Sommerlad A, et al. Dementia prevention, intervention, and care: 2020 report of the Lancet Commission. Lancet. 2020;396(10248):413-446. DOI: https: / / doi.org/10.1016/S0140-6736(20)30367-6

16. Ngandu T, Lehtisalo J, Solomon A, et al. A 2 year multidomain intervention of diet, exercise, cognitive training, and vascular risk monitoring versus control to prevent cognitive decline in at-risk elderly people (FINGER): a randomised controlled trial. Lancet. 2015;385(9984):2255-2263. DOI: https: / / doi.org/10.1016/S0140-6736(15)60461-5

17. Walker KA, Ficek BN, Westbrook R. Understanding the Role of Systemic Inflammation in Alzheimer's Disease. ACS Chem Neurosci. 2019;10(8):33403342. DOI: https:/ / doi.org/10.1021/acschemneuro.9b00333

18. Kuzma E, Lourida I, Moore SF, Levine DA, Ukoumunne OC, Llewellyn DJ. Stroke and dementia risk: A systematic review and meta-analysis. Alzheimers Dement. 2018;14(11):1416-1426. DOI: https://doi.org/10.1016/j. jalz.2018.06.3061

19. Ou YN, Tan CC, Shen XN, et al. Blood Pressure and Risks of Cognitive Impairment and Dementia: A Systematic Review and Meta-Analysis of 209 Prospective Studies. Hypertension. 2020;76(1):217-225. DOI: https:/ / doi. org / 10.1161/HYPERTENSIONAHA.120.14993

20. Rait G, Walters K, Bottomley C, et al. Survival of people with clinical diagnosis of dementia in primary care: cohort study. BMJ. 2010;341:c3584. DOI: https:/ / doi.org/10.1136/bmj.c3584

21. Pham TM, Petersen I, Walters $\mathrm{K}$, et al. Trends in dementia diagnosis rates in UK ethnic groups: analysis of UK primary care data. Clin Epidemiol. 2018;10:949-960. DOI: https:/ / doi.org/10.2147/CLEP.S152647

22. Rochoy M, Rivas V, Chazard E, et al. Factors Associated with Alzheimer's Disease: An Overview of Reviews. J Prev Alzheimers Dis. 2019;6(2):121-134. DOI: https: / / doi.org/10.14283/jpad.2019.7

23. Ng TP, Lee TS, Lim WS, et al. Development, Validation and Field Evaluation of the Singapore Longitudinal Ageing Study (SLAS) Risk Index for Prediction of Mild Cognitive Impairment and Dementia. J Prev Alzheimers Dis. 2021;8(3):335-344. DOI: https:/ / doi.org/10.14283/jpad.2021.19

24. Srikanth V, Sinclair AJ, Hill-Briggs F, Moran C, Biessels GJ. Type 2 diabetes and cognitive dysfunction-towards effective management of both comorbidities. Lancet Diabetes Endocrinol. 2020;8(6):535-545. DOI: https://doi.org/10.1016/ S2213-8587(20)30118-2

25. Donegan K, Fox N, Black N, Livingston G, Banerjee S, Burns A. Trends in diagnosis and treatment for people with dementia in the UK from 2005 to 2015: a longitudinal retrospective cohort study. Lancet Public Health 2017;2(3):e149-e156. DOI: http:/ / dx.doi.org/10.1016/S2468-2667(17)30031-2

26. Wang K, Liu H. Early-Onset Subgroup of Type 2 Diabetes and Risk of Dementia, Alzheimer's disease and Stroke: A Cohort Study. J Prev Alzheimers Dis. 2021;4(8):442-447. DOI: https: / / doi.org/10.14283/jpad.2021.35

27. Gauthier S, Albert M, Fox N, et al. Why has therapy development for dementia failed in the last two decades? Alzheimers Dement. 2016;12(1):60-64. DOI: https: / / doi.org/10.1016/j.jalz.2015.12.003

How to cite this article: B. Zheng, B. Su, C. Udeh-Momoh, et al. Associations of Cardiovascular and Non-Cardiovascular Comorbidities with Dementia Risk in Patients with Diabetes: Results from a Large UK Cohort Study. J Prev Alz Dis 2022;1(9):86-91, http: / / dx.doi.org/10.14283/jpad.2022.8 